\title{
CALDERÓN-ZYGMUND OPERATORS ASSOCIATED TO ULTRASPHERICAL EXPANSIONS
}

\author{
DARIUSZ BURACZEWSKI, TERESA MARTINEZ, AND JOSÉ L. TORREA
}

\begin{abstract}
We define the higher order Riesz transforms and the LittlewoodPaley $g$-function associated to the differential operator $L_{\lambda} f(\theta)=-f^{\prime \prime}(\theta)-$ $2 \lambda \cot \theta f^{\prime}(\theta)+\lambda^{2} f(\theta)$. We prove that these operators are Calderón-Zygmund operators in the homogeneous type space $\left((0, \pi),(\sin t)^{2 \lambda} d t\right)$. Consequently, $L^{p}$ weighted, $H^{1}-L^{1}$ and $L^{\infty}-B M O$ inequalities are obtained.
\end{abstract}

intro

\section{INTRODUCTION}

B. Muckenhoupt and E. Stein, in $[$ MS , defined and studied the versions of some objects to classical Fourier Analysis (conjugate functions, maximal functions, g-functions and multipliers) for the system of the ultraspherical polynomials. Their approach follows the lines of the classical Fourier Analysis in the torus. In particular, the relationship between Fourier series, analytic functions and harmonic functions plays an essential role. For instance, their definition of the conjugate function was via a boundary value limit of certain "conjugate harmonic function" which satisfies the appropriate Cauchy-Riemann equations. The technique involved the definition of the "harmonic extension" including a careful analysis of its kernel. Then they built a conjugate harmonic function and proved the existence of a boundary value function, the conjugate function. They got $L^{p}$ boundedness for $p$ in the range $1<p<\infty$ and some substitutive inequality in the case $p=1$. This method was followed later by different authors when defining classical operators for orthogonal expansions. In $\frac{75}{5}$ they did not study the kernel of the conjugate function.

Five years later, there appeared Stein's celebrated monograph st]1, where "maximal functions", " $g$-functions", "Riesz transforms", and "multipliers" were also defined. He used systematically a point of view based on an analysis of a Laplacian on compact Lie groups. He studied the "heat" and "Poisson" semigroups associated to the Laplacian and, from them, he derived the rest of the operators by using some spectral formulas.

Date: March 13, 2005.

2000 Mathematics Subject Classification. 42C05,42C15.

Key words and phrases. Ultraspherical polynomials, Calderón-Zygmund operators.

The authors were partially supported by RTN Harmonic Analysis and Related Problems contract HPRN-CT-2001-00273-HARP. First author was supported in part by KBN grant 1P03A01826. Second and third authors were partially supported by BFM grant 2002-04013C02-02. 
A natural question arising from $\left.\frac{\text { MS }}{5}\right]$ is studying some other classical operators in the context of the system of the ultraspherical polynomials. In particular, our aim was to study higher order Riesz transforms. In order to define those operators, it seems that the natural procedure to follow is the one suggested in the monograph [7], and we do so in this paper. The method used to prove the boundedness of higher order Riesz transforms could be applied to a more general class of operators. For instance, we present in this paper its application to study the boundedness of the Littlewood-Paley $g$-function, although more operators fit in this technique (e.g. multipliers of Laplace transform type). More concretely, we find that all these operators are naturally Calderón-Zygmund operators in a space of homogeneous type. Therefore, we get as a byproduct of the general theory $L^{p}, H^{1}, B M O$ boundedness and weighted inequalities for them (see Theorem 1.4).

${ }_{B M Y} B_{y}$ using a different method $d_{s t 1}$ the (first order) Riesz transform was studied in 1]. It was defined following [7] and, among other results, the $L^{p}$-boundedness for $p \in(1, \infty)$ and the weak type $(1,1)$ were obtained.

We consider the ultraspherical polynomials $P_{n}^{\lambda}(x), \lambda>0$, defined as the coefficients in the expansion of the generating function $\left(1-2 x \omega+\omega^{2}\right)^{-\lambda}=$ $\sum_{n=0}^{\infty} \omega^{n} P_{n}^{\lambda}(x)$ (see [8] for further details). It is known that the set $\left\{P_{n}^{\lambda}(\cos \theta)\right.$ : $n \in \mathbb{N}\}$ is orthogonal and complete in $L^{2}[0, \pi]$ with respect to the measure $d m_{\lambda}(\theta)=(\sin \theta)^{2 \lambda} d \theta$. The functions $P_{n}^{\lambda}(\cos \theta)$ are eigenfunctions of the operator $L_{\lambda}$

$$
L_{\lambda} f(\theta)=-f^{\prime \prime}(\theta)-2 \lambda \cot \theta f^{\prime}(\theta)+\lambda^{2} f(\theta),
$$

with eigenvalues $\mu_{n}=(n+\lambda)^{2}$. Every function $f$ in $L^{2}\left(d m_{\lambda}\right)$ has the ultraspherical expansion $f(\theta)=\sum_{n=0}^{\infty} a_{n} P_{n}^{\lambda}(\cos \theta)\left\|P_{n}^{\lambda}\right\|_{L^{2}\left(d m_{\lambda}\right)}^{-1}$. Following [t], we define its Poisson integral as

$$
P f\left(e^{-t}, \theta\right)=e^{-t \sqrt{L_{\lambda}}} f(\theta)=\sum_{n=0}^{\infty} a_{n} e^{-t(n+\lambda)} P_{n}^{\lambda}(\cos \theta)\left\|P_{n}^{\lambda}\right\|_{L^{2}\left(d m_{\lambda}\right)}^{-1}
$$

The calculus formula $s^{-a}=\frac{1}{\Gamma(a)} \int_{0}^{\infty} e^{-t s} t^{a-1} d t$ can be used to define

tion Llambda-05

$$
L_{\lambda}^{-l / 2} f(\theta)=\frac{1}{\Gamma(l)} \int_{0}^{\infty} e^{-t \sqrt{L_{\lambda}}} f(\theta) t^{l-1} d t, \quad l \geq 1, \quad f \in L^{2}\left(d m_{\lambda}\right) .
$$

On the other hand, it is easy to check that $L_{\lambda}$ is formally self-adjoint on the space $L^{2}\left(d m_{\lambda}\right)$ and that it factorizes as $L_{\lambda} f(\theta)=\left(-\partial_{\theta}^{*} \partial_{\theta}+\lambda^{2}\right) f(\theta)$, where $\partial_{\theta}^{*}=\partial_{\theta}+2 \lambda$ st $\cot \theta$ is formally adjoint to $\partial_{\theta}$, i.e., $\left\langle\partial_{\theta}^{*} f, g\right\rangle_{L^{2}\left(m_{\lambda}\right)}=-\left\langle f, \partial_{\theta} g\right\rangle_{L^{2}\left(m_{\lambda}\right)}$. Following [7], the Riesz transform $(l=1)$ and the higher order Riesz transforms $(l>1)$ are defined as

$$
R_{\lambda}^{l} f(\theta)=\partial_{\theta}^{l}\left(L_{\lambda}\right)^{-l / 2} f(\theta), \quad l \geq 1
$$


Also, the Littlewood-Paley $g$-function is defined as

$$
\mathcal{G} f(\theta)=\left(\int_{0}^{1} r \log \frac{1}{r}\left(\left|\partial_{r} P f(r, \theta)\right|^{2}+\left|\partial_{\theta} \operatorname{Pf}(r, \theta)\right|^{2}\right) d r\right)^{1 / 2} .
$$

For these operators, we have the following result.

eorem principal

orincipal Riesz

eq $\mathrm{g} 1$

eq g2

rem principal g

eq polynomial

echnical lemmas
Theorem 1.4. The operators $R_{\lambda}^{l} f$, for any natural $l \geq 1$, and $\mathcal{G} f$ are bounded in $L^{p}\left(w d m_{\lambda}\right), 1<p<\infty$ for any weight $w$ in the Muckenhoupt class $A_{p}$ with respect to the measure $d m_{\lambda}$ and of weak type $(1,1)$ with respect to the measure $w d m_{\lambda}$ for $w \in A_{1}$. Also, they map $L^{\infty}$ into $B M O\left(d m_{\lambda}\right)$ and $H^{1}\left(d m_{\lambda}\right)$ into $L^{1}\left(d m_{\lambda}\right)$ boundedly.

In fact, this theorem is a consequence of the general theory for CalderónZygmund operators in spaces of homogeneous type and the following theorems.

Theorem 1.5. For any natural $l \geq 1$, the operators $R_{\lambda}^{l}$ are Calderón-Zygmund operators in the homogeneous type space $\left([0, \pi), d m_{\lambda}\right)$.

For the $g$-function, we study separately the operators

$$
\begin{aligned}
& \mathcal{G}^{1} f(\theta)=\left(\int_{0}^{1} r \log \frac{1}{r}\left|\partial_{r} P_{r} f(\theta)\right|^{2} d r\right)^{1 / 2} \\
& \mathcal{G}^{2} f(\theta)=\left(\int_{0}^{1} r \log \frac{1}{r}\left|\partial_{\theta} P_{r} f(\theta)\right|^{2} d r\right)^{1 / 2} .
\end{aligned}
$$

Theorem 1.8. There exist vector valued Calderón-Zygmund operators in the homogeneous type space $\left([0, \pi), d m_{\lambda}\right)$ mapping scalar-valued functions into functions with values in $L^{2}((0,1), d r), T_{\mathcal{G}^{i}}, i=1,2$, such that

$$
\mathcal{G}^{i} f(\theta)=\left\|T_{\mathcal{G}^{i}} f(\theta)\right\|_{L^{2}((0,1), d r)} .
$$

The structure of the paper is as follows. In section 2, we state the technical Theorem 2.4, as an intermediate step in the proofs of Theorems 1.5 and 1.8. In section 3 we verify the hypothesis of Theorem 2.4 for the higher order Riesz transforms, and in section 4 we do the corresponding checking for the $g$-function.

Let us mention just a word about notation. Throughout the paper, the letter $C$ will denote a constant whose value may vary from line to line, and let us call polynomial function to any finite linear combination of ultraspherical polynomials, that is, any $f$ of the form

$$
f=\sum_{n=0}^{N} \frac{a_{n} P_{n}^{\lambda}}{\left\|P_{n}^{\lambda}\right\|_{L^{2}\left(d m_{\lambda}\right)}}, \quad a_{n}=\int_{0}^{\pi} f(\theta) \frac{P_{n}^{\lambda}(\cos \theta)}{\left\|P_{n}^{\lambda}\right\|_{L^{2}\left(d m_{\lambda}\right)}} d m_{\lambda}(\theta)
$$

\section{PRELIMinaries AND TECHNiCAL TOOLS}

Following [3] and [W], a space of homogeneus type $(X, \rho, \mu)$ is a set $X$ together with a quasimetric $\rho$ and a positive measure $\mu$ on $X$ such that for every $\theta \in X$ and $r>0, \mu(B(\theta, r))<\infty$, and such that there exists $0<C<\infty$ such that 
for every $\theta \in X$ and $r>0 \mu(B(\theta, 2 r)) \leq C \mu(B(\theta, r))$. In our case, $X=[0, \pi]$ with the metric given by the absolute value, and the measure is $d m_{\lambda}$, which is doubling, what can be easily checked.

We say that a kernel $K: X \times X \backslash\{x=y\} \rightarrow \mathbb{C}$ is a standard kernel if there exist $\varepsilon>0$ and $C<\infty$ such that for all $x \neq y \in X$ and $z$ with $\rho(x, z) \leq \varepsilon \rho(x, y)$,

ion CZ kernel 1

ion CZ kernel 2

$$
\begin{aligned}
& |K(x, y)| \leq \frac{C}{\mu(B(x, r))}, \quad \text { where } \quad r=\rho(x, y), \quad \text { and } \\
& |K(x, y)-K(z, y)|+|K(y, x)-K(y, z)| \leq \frac{\rho(x, z)}{\rho(x, y)} \frac{C}{\mu(B(x, r))} .
\end{aligned}
$$

Thus, a Calderón-Zygmund operator (with associated kernel $K$ ) is a linear operator $T$ bounded in $L^{2}$ such that, for every $f \in L^{2}$ and $x$ outside the support of $f$,

$$
T f(x)=\int_{X} K(x, y) f(y) d \mu(y) .
$$

It is known that any Calderón-Zygmund operator as above is bounded in $L^{p}(w d \mu)$, for $1<p<\infty$ and any weight $w$ in the Muckenhoupt class $A_{p}$ with respect to the measure $d \mu$. They also map $L^{1}(w d \mu)$ into $L^{1, \infty}(w d \mu)$ for any weight $w$ in the Muckenhoupt class $A_{1}$ with respect to the measure $d \mu$, $L^{\infty}(\mu)$ boundedly into $B M O(d \mu)$ and $H^{1}(d \mu)$ into $L^{1}(d \mu)$ (see [2]).

For $\mathbb{B}$ a Banach space, vector-valued Calderón-Zygmund operators, $T$ from $L^{2}(d \mu)$ into $L_{\mathbb{B}}^{2}(d \mu)$, are defined in the same way as scalar valued ones, but considering $K: X \times X \backslash\{x=y\} \rightarrow \mathbb{B}$ instead of a scalar valued kernel, and taking $\mathbb{B}$-norms in (2.1) and (2.2) instead of absolute values. The boundedness results mentioned above also hold in the vector valued case (see $[6]$ ).

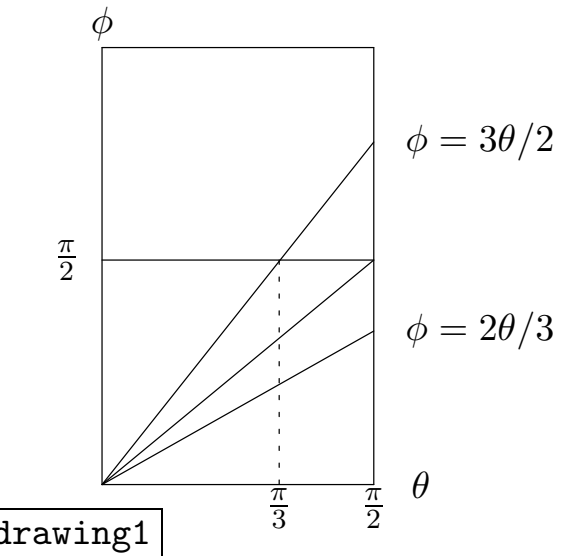

The symmetry with respect to $\pi / 2$ in the kernels of our operators will play an important role in the proofs. Also, it will be useful to have in mind the picture of the area where we are placing the variables $\theta$ and $\phi$. Our first step is studying in detail the behavior of the measure of $B(\theta,|\theta-\phi|)$.

Lemma 2.3. There exists a constant $C>0$ such that for any $\theta \in[0, \pi / 2]$,

$$
m_{\lambda}(B(\theta,|\theta-\phi|)) \leq C \begin{cases}|\theta-\phi|(\sin (\theta \vee \phi))^{2 \lambda} & \text { if } \phi \in[0, \pi / 2] \\ \phi & \text { if } \phi \in[\pi / 2, \pi], \phi>\frac{3}{2} \theta \\ |\theta-\phi|(\sin (\theta \vee \phi))^{2 \lambda} & \text { if } \phi \in[\pi / 2, \pi], \phi<\frac{3}{2} \theta\end{cases}
$$


ger conditions

strong kernel 1

strong kernel 2
Theorem 2.4. Let $\mathbb{B}$ be a Banach space, and $T: L^{2}\left(d m_{\lambda}\right) \rightarrow L_{\mathbb{B}}^{2}\left(d m_{\lambda}\right)$ be an operator given by integration against a kernel $K$ in the Calderón-Zygmund sense, such that

i) If $|\theta-\phi| \geq \pi / 6$, then

$$
\|K(\theta, \phi)\|_{\mathbb{B}} \leq C
$$

and

$$
\left\|\partial_{\theta} K(\theta, \phi)\right\|_{\mathbb{B}}+\left\|\partial_{\phi} K(\theta, \phi)\right\|_{\mathbb{B}} \leq C .
$$

ii) For every $(\theta, \phi)$ either belonging to $[0, \pi / 2] \times[0, \pi / 2]$ or to $[0, \pi / 2] \times$ $[\pi / 2, \pi] \cap\left\{\phi \leq \frac{3}{2} \theta\right\}$, we have

$$
\begin{aligned}
& \|K(\theta, \phi)\|_{\mathbb{B}} \leq \frac{C}{|\theta-\phi|} \frac{1}{(\sin (\theta \vee \phi))^{2 \lambda}}, \quad \text { and } \\
& \left\|\partial_{\theta} K(\theta, \phi)\right\|_{\mathbb{B}}+\left\|\partial_{\phi} K(\theta, \phi)\right\|_{\mathbb{B}} \leq \frac{C}{|\theta-\phi|^{2}} \frac{1}{(\sin (\theta \vee \phi))^{2 \lambda}}
\end{aligned}
$$

iii) The kernel $K$ is symmetric $(K(\pi-\theta, \pi-\phi)=K(\theta, \phi))$ or antisymmetric $(K(\pi-\theta, \pi-\phi)=-K(\theta, \phi))$.

Then, $T$ is a Calderón-Zygmund operator in $(0, \pi)$ with $\rho(x, y)=|x-y|$.

ProOF. Lemma 2.3 and (2.5) clearly imply (2.1) in the region stated in ii). On the other hand, for $(\theta, \phi) \in[0, \pi / 2] \times[\pi / 2, \pi] \cap\left\{\phi \geq \frac{3}{2} \theta\right\}$, we have $\pi / 6 \leq$ $|\theta-\phi| \leq \pi$, and therefore, by i), $\|K(\theta, \phi)\|_{\mathbb{B}} \leq C \leq \frac{C \pi}{\phi}$. Using again Lemma $2.3,(2.5)$ and the symmetry condition iii), we obtain (2.1) easily.

By using Lemma 2.3 and (2.6), we get

$$
\left\|\partial_{\theta} K(\theta, \phi)\right\|_{\mathbb{B}}+\left\|\partial_{\phi} K(\theta, \phi)\right\|_{\mathbb{B}} \leq \frac{C}{|\theta-\phi|} \frac{1}{m_{\lambda}(B(\theta,|\theta-\phi|))}
$$

for $\theta$ and $\phi$ in the region stated in ii). For $(\theta, \phi) \in[0, \pi / 2] \times[\pi / 2, \pi] \cap\left\{\phi \geq \frac{3}{2} \theta\right\}$, we have $\pi / 6 \leq|\theta-\phi| \leq \pi$, and therefore, by i), $\left\|\partial_{\theta} K(\theta, \phi)\right\|_{\mathbb{B}}+\left\|\partial_{\phi} K(\theta, \phi)\right\|_{\mathbb{B}} \leq$ $C \leq \frac{C}{|\theta-\phi| \phi}$. By Lemma 2.3, we have $(2.7)$ in $(\theta, \phi) \in[0, \pi / 2] \times[\pi / 2, \pi] \cap\{\phi \geq$ $\left.\frac{3}{2} \theta\right\}$. The symmetry condition iii) implies that $(2.7)$ holds for any $(\theta, \phi) \in$ $[0, \pi] \times[0, \pi]$. By standard calculations, this inequality implies condition (2.2) for $\varepsilon=1 / 2$

\section{Proof of Theorem 1.5}

Given a polynomial function $f$, we have

$$
\begin{aligned}
R_{\lambda}^{l} f(\theta) & =\partial_{\theta}^{l}\left(L_{\lambda}\right)^{-l / 2} f(\theta)=\partial_{\theta}^{l}\left(\sum_{n=0}^{N} \frac{a_{n}}{(n+\lambda)^{k}} \frac{P_{n}^{\lambda}(\cos \theta)}{\left\|P_{n}^{\lambda}\right\|_{L^{2}\left(d m_{\lambda}\right)}}\right) \\
& =\sum_{n=0}^{N} \frac{a_{n}}{(n+\lambda)^{l}} \frac{\partial_{\theta}^{l} P_{n}^{\lambda}(\cos \theta)}{\left\|P_{n}^{\lambda}\right\|_{L^{2}\left(d m_{\lambda}\right)}}
\end{aligned}
$$


The case $l=1$ was extensively studied in $\frac{\text { BMTU }}{1] .}$ There it was proved its boundedness in $L^{p}$ for $p \in(1, \infty)$ and its weak type $(1,1)$, as well as the fact that it is a principal value, the boundedness of the maximal operator, etc.. In order to prove Theorem 1.5, we first prove the boundedness in $L^{2}$ of the higher order Riesz transforms.

osection 12 bdd

differential

riesz2 operator

riesz operator

eq fundamental
3.1. $L^{2}$ boundedness of higher order Riesz transforms. We shall see that for any polynomial function (1.9), we have $\left\|R_{\lambda}^{l} f\right\|_{L^{2}\left(d m_{\lambda}\right)} \leq C\|f\|_{L^{2}\left(d m_{\lambda}\right)}$ with a constant independent of $N$. First of all, let us observe that without loss of generality, we can consider only polynomial functions $f$ such that there only appear $P_{n}^{\lambda}(\cos \theta)$ for $n \geq l+1$ in their expansion. Since any linear operator is bounded on finite dimensional spaces, we have that the restriction of $R_{\lambda}^{l}$ to the span of $\left\{P_{0}^{\lambda}, \ldots, P_{l}^{\lambda}\right\}$ is bounded, and it only remains to check the case when $n \geq l+1$.

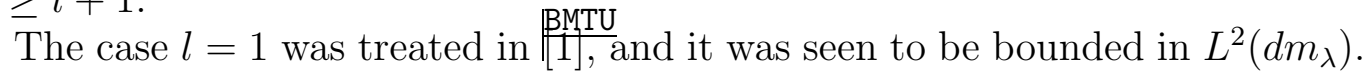
Lets us explore the case $l=2$. In this case, for any $n \geq 3$

$$
\begin{aligned}
R_{\lambda}^{2} P_{n}^{\lambda}(\cos \theta) & =\frac{1}{(n+\lambda)^{2}} \partial_{\theta}^{2} P_{n}^{\lambda}(\cos \theta) \\
& =\frac{-2 \lambda}{(n+\lambda)^{2}} \cos \theta P_{n-1}^{\lambda+1}(\cos \theta)+\frac{4 \lambda(\lambda+1)}{(n+\lambda)^{2}}(\sin \theta)^{2} P_{n-2}^{\lambda+2}(\cos \theta) .
\end{aligned}
$$

On the other hand, from (1.1) we obtain $\partial_{\theta}^{2}=-L_{\lambda}-2 \lambda \cot \theta \partial_{\theta}+\lambda^{2}$ and therefore

$$
R_{\lambda}^{2}=\partial_{\theta}^{2} L_{\lambda}^{-1}=-\mathrm{Id}-2 \lambda \cot \theta \partial_{\theta} L_{\lambda}^{-1}+\lambda^{2} L_{\lambda}^{-1}
$$

With this expression,

$$
R_{\lambda}^{2} P_{n}^{\lambda}=-P_{n}^{\lambda}+\frac{4 \lambda^{2}}{(n+\lambda)^{2}} \cos \theta P_{n-1}^{\lambda+1}+\frac{\lambda^{2}}{(n+\lambda)^{2}} P_{n}^{\lambda} .
$$

Mixing (3.2) and (3.4) one has

$$
\begin{aligned}
& \frac{2 \lambda(2 \lambda+1)}{(n+\lambda)^{2}} \cos \theta P_{n-1}^{\lambda+1}(\cos \theta) \\
& =P_{n}^{\lambda}(\cos \theta)-\frac{\lambda^{2}}{(n+\lambda)^{2}} P_{n}^{\lambda}(\cos \theta)+\frac{4 \lambda(\lambda+1)}{(n+\lambda)^{2}}(\sin \theta)^{2} P_{n-2}^{\lambda+2}(\cos \theta) .
\end{aligned}
$$

In particular, with this calculation we get from (3.4)

$$
\begin{aligned}
R_{\lambda}^{2} P_{n}^{\lambda}(\cos \theta)= & \frac{-1}{2 \lambda+1} P_{n}^{\lambda}(\cos \theta)+\frac{1}{2 \lambda+1} \frac{1}{(n+\lambda)^{2}} P_{n}^{\lambda}(\cos \theta) \\
& +\frac{8 \lambda^{2}(\lambda+1)}{2 \lambda+1} \frac{1}{(n+\lambda)^{2}}(\sin \theta)^{2} P_{n-2}^{\lambda+2}(\cos \theta)
\end{aligned}
$$


and therefore

$$
\begin{aligned}
R_{\lambda}^{2} f(\theta)= & \frac{-1}{2 \lambda+1} \sum_{n=3}^{N} \frac{a_{n}}{\left\|P_{n}^{\lambda}\right\|_{L^{2}\left(d m_{\lambda}\right)}} P_{n}^{\lambda}(\cos \theta) \\
& +\frac{1}{2 \lambda+1} \sum_{n=3}^{N} \frac{a_{n}}{\left\|P_{n}^{\lambda}\right\|_{L^{2}\left(d m_{\lambda}\right)}} \frac{1}{(n+\lambda)^{2}} P_{n}^{\lambda}(\cos \theta) \\
& +\frac{8 \lambda^{2}(\lambda+1)}{2 \lambda+1} \sum_{n=3}^{N} \frac{a_{n}}{\left\|P_{n}^{\lambda}\right\|_{L^{2}\left(d m_{\lambda}\right)}} \frac{1}{(n+\lambda)^{2}}(\sin \theta)^{2} P_{n-2}^{\lambda+2}(\cos \theta)
\end{aligned}
$$

Clearly, the first two sums are operators bounded in $L^{2}\left(d m_{\lambda}\right)$. For the third one, we will use the following lemma.

Lemma 3.6. For every $k \geq 0$, the operator $T$ acting on polynomial functions

$$
T f(\theta)=\sum_{n=k+1}^{N} \frac{a_{n}}{\left\|P_{n}^{\lambda}\right\|_{L^{2}\left(d m_{\lambda}\right)}} \frac{1}{(n+\lambda)^{k}}(\sin \theta)^{k} P_{n-k}^{\lambda+k}(\cos \theta)
$$

is bounded in $L^{2}\left(d m_{\lambda}\right)$.

Proof. By using the orthogonality of $(\sin \theta)^{k} P_{n-k}^{\lambda+k}$ in $L^{2}\left(d m_{\lambda}\right)$, and

$$
\left\|P_{n}^{\lambda}\right\|_{L^{2}\left(d m_{\lambda}\right)}^{2}=2^{1-2 \lambda} \pi \Gamma(\lambda)^{-2} \frac{\Gamma(n+2 \lambda)}{(n+\lambda) n !},
$$

(see [8]), we obtain

$$
\begin{aligned}
& \left\|\sum_{n=k+1}^{N} \frac{a_{n}}{\left\|P_{n}^{\lambda}\right\|_{L^{2}\left(d m_{\lambda}\right)}} \frac{1}{(n+\lambda)^{k}}(\sin \theta)^{k} P_{n-k}^{\lambda+k}\right\|_{L^{2}\left(d m_{\lambda}\right)}^{2} \\
& =\sum_{n=k+1}^{N} \frac{a_{n}^{2}}{\left\|P_{n}^{\lambda}\right\|_{L^{2}\left(d m_{\lambda}\right)}^{2}} \frac{\left\|P_{n-k}^{\lambda+k}\right\|_{L^{2}\left(d m_{\lambda+k}\right)}^{2}}{(n+\lambda)^{2 k}} \leq C \sum_{n=k+1}^{N} a_{n}^{2}=C\|f\|_{L^{2}\left(d m_{\lambda}\right)}^{2} .
\end{aligned}
$$

We use induction in $l$ to prove the boundedness of $R_{\lambda}^{l}$ for any $l \geq 1$. Let us assume that $R_{\lambda}^{k}$ is bounded in $L^{2}\left(d m_{\lambda}\right)$ for $k \leq l-1$. ¿From (3.3), for $l \geq 3$, we have

riesz operator

$$
\begin{aligned}
R_{\lambda}^{l} & =\partial_{\theta}^{l} L_{\lambda}^{-l / 2}=\partial_{\theta}^{l-2} R_{\lambda}^{2} L_{\lambda}^{-(l-2) / 2} \\
& =-\partial_{\theta}^{l-2} L_{\lambda}^{-(l-2) / 2}-2 \lambda \partial_{\theta}^{l-2} \cot \theta \partial_{\theta} L_{\lambda}^{-l / 2}+\lambda^{2} \partial_{\theta}^{l-2} L_{\lambda}^{-l / 2} \\
& =-R_{\lambda}^{l-2}-2 \lambda \partial_{\theta}^{l-2} \cot \theta \partial_{\theta} L_{\lambda}^{-l / 2}+\lambda^{2} R_{\lambda}^{l-2} L_{\lambda}^{-1} .
\end{aligned}
$$

By the induction hypothesis, first and last operators are bounded in $L^{2}\left(d m_{\lambda}\right)$. It remains to show that the second term is also bounded. 
Lemma 3.8. For any $k \geq 0$,

$$
\partial_{\theta}^{k}\left(\cos \theta P_{n-1}^{\lambda+1}(\cos \theta)\right)=\sum_{a=1}^{k+1} \sum_{\substack{A, B: \\ A+B=a}} C_{A B}(\cos \theta)^{A}(\sin \theta)^{B} P_{n-a}^{\lambda+a}(\cos \theta)
$$

where $C_{A B} \in \mathbb{R}$ may be zero.

ProOF. We will prove the result by induction in $k$. For $k=0$ it is clearly true, with $C_{10}=1$ and $C_{01}=0$. Let us suppose that the formula holds until $k-1$. For $k$, we have

$$
\partial_{\theta}^{k}\left(\cos \theta P_{n-1}^{\lambda+1}(\cos \theta)\right)=\partial_{\theta} \sum_{a=1}^{k} \sum_{\substack{A, B: \\ A+B=a}} C_{A B}(\cos \theta)^{A}(\sin \theta)^{B} P_{n-a}^{\lambda+a}(\cos \theta)
$$

and it is enough to see that any term $\partial_{\theta}\left((\cos \theta)^{A}(\sin \theta)^{B} P_{n-a}^{\lambda+a}(\cos \theta)\right)$ is a sum of terms of the form $(\cos \theta)^{\tilde{A}}(\sin \theta)^{\tilde{B}} P_{n-\tilde{a}}^{\lambda+\tilde{a}}(\cos \theta) \operatorname{con} 1 \leq \tilde{a} \leq k+1, \tilde{A}+\tilde{B}=\tilde{a}$. And this holds, since

$$
\begin{aligned}
\partial_{\theta}\left((\cos \theta)^{A}(\sin \theta)^{B} P_{n-a}^{\lambda+a}(\cos \theta)\right) & =A(\cos \theta)^{A-1}(\sin \theta)^{B+1} P_{n-a}^{\lambda+a}(\cos \theta) \\
& +B(\cos \theta)^{A+1}(\sin \theta)^{B-1} P_{n-a}^{\lambda+a}(\cos \theta) \\
& -2(\lambda+a)(\cos \theta)^{A}(\sin \theta)^{B+1} P_{n-(a+1)}^{\lambda+(a+1)}(\cos \theta) .
\end{aligned}
$$

Thus, for a polynomial function $f=\sum_{n=l+1}^{N} a_{n}\left\|P_{n}^{\lambda}\right\|_{L^{2}\left(d m_{\lambda}\right)}^{-1} P_{n}^{\lambda}$, we can write $\partial_{\theta}^{l-2} \cot \theta \partial_{\theta} L_{\lambda}^{-l / 2} f(\theta)=\sum_{n=l+1}^{N} \frac{a_{n}}{\left\|P_{n}^{\lambda}\right\|_{L^{2}\left(d m_{\lambda}\right)}} \frac{1}{(n+\lambda)^{l}} \partial_{\theta}^{l-2}\left(-2 \lambda \cos \theta P_{n-1}^{\lambda+1}(\cos \theta)\right)$

$$
\begin{aligned}
& =\sum_{a=1}^{l-1} \sum_{\substack{A, B: \\
A+B=a}} C_{A B} \sum_{n=l+1}^{N} \frac{a_{n}}{\left\|P_{n}^{\lambda}\right\|_{L^{2}\left(d m_{\lambda}\right)}} \frac{1}{(n+\lambda)^{l}}(\cos \theta)^{A}(\sin \theta)^{B} P_{n-a}^{\lambda+a}(\cos \theta) \\
& =\sum_{a=1}^{l-1} \sum_{\substack{A, B: \\
A+B=a}} C_{A B} T_{A, B, a}^{l-1} f(\theta) .
\end{aligned}
$$

Thus, the boundedness of this operator is done with the following lemma.

oundedness TABa Lemma 3.10. For any $l \geq 2$, the operators $T_{A, B, a}^{l-1}$ appearing in (3.9) with non zero coefficients are bounded in $L^{2}\left(d m_{\lambda}\right)$.

Proof. We will proceed by induction in $A$ : in the case $A=0$, we have to prove that for every $a \leq l-1$,

$$
T_{0, a a}^{l-1} f(\theta)=\sum_{n=l+1}^{N} \frac{a_{n}}{\left\|P_{n}^{\lambda}\right\|_{L^{2}\left(d m_{\lambda}\right)}} \frac{1}{(n+\lambda)^{l}} \cos \theta(\sin \theta)^{a} P_{n-a}^{\lambda+a}(\cos \theta)
$$


is bounded in $L^{2}\left(d m_{\lambda}\right)$. This holds by Lemma 3.6. Now assume that the induction hypothesis is true until $A-1$, that is, for any $\tilde{A} \leq A-1$, we have that for every $a \leq l-1$, the operator $T_{\tilde{A}, a-\tilde{A}, a}^{l-1} f(\theta)$ is bounded in $L^{2}\left(d m_{\lambda}\right)$. For $A \geq 1$, by (3.5) with $n-(a-1)$ and $\lambda+(a-1)$ instead of $n$ and $\lambda$, one can write

$$
\begin{gathered}
\frac{1}{(n+\lambda)^{l}}(\cos \theta)^{A}(\sin \theta)^{B} P_{n-a}^{\lambda+a}=\frac{C}{(n+\lambda)^{l-2}}(\cos \theta)^{A-1}(\sin \theta)^{B} \times \\
\times\left(P_{n-(a-1)}^{\lambda+(a-1)}+\frac{C}{(n+\lambda)^{2}} P_{n-(a-1)}^{\lambda+(a-1)}+\frac{C}{(n+\lambda)^{2}}(\sin \theta)^{2} P_{n-(a+1)}^{\lambda+(a+1)}\right) \\
=\frac{C}{(n+\lambda)^{l-2}}(\cos \theta)^{A-1}(\sin \theta)^{B} P_{n-(a-1)}^{\lambda+(a-1)}+\frac{C}{(n+\lambda)^{l}}(\cos \theta)^{A-1}(\sin \theta)^{B} P_{n-(a-1)}^{\lambda+(a-1)} \\
\quad+\frac{C}{(n+\lambda)^{l}}(\cos \theta)^{A-1}(\sin \theta)^{B+2} P_{n-(a+1)}^{\lambda+(a+1)} .
\end{gathered}
$$

The first two terms give rise to operators bounded in $L^{2}\left(d m_{\lambda}\right)$, by the induction hypothesis. For the third factor, if $a+1 \leq l-1$, then by the same reason it is also a bounded operator. But we want to prove the boundedness for any $a \leq l-1$, and thus it remains to prove the case $a=l-1$. Since $\partial_{\theta}^{l-2} \cot \theta \partial_{\theta} L_{\lambda}^{-l / 2} P_{n}^{\lambda}=$ $-2 \lambda \partial_{\theta}^{l-2}\left(\cos \theta P_{n-1}^{\lambda+1}\right)$, an operator $T_{A, B, l-1}^{l-1}$ in which there appear terms with $P_{n-(l-1)}^{\lambda+(l-1)}$ in the left-hand of expression (3.9), necessarily comes from the factors in which the derivatives $\partial_{\theta}^{l-2}$ act on the polynomial $P_{n-1}^{\lambda+1}$ and not in any other term (otherwise we would obtain $P_{n-a}^{\lambda+a}$ with $a<l-1$ ). Therefore, the only terms with non-zero coefficients coming by applying (3.5) in the right-hand of (3.9) must sum up a constant times the operator

$$
T_{1, l-2, l-1}^{l-1} f(\theta)=\sum_{n=l+1}^{N} \frac{a_{n}}{\left\|P_{n}^{\lambda}\right\|_{L^{2}\left(d m_{\lambda}\right)}} \frac{1}{(n+\lambda)^{l}} \cos \theta(\sin \theta)^{l-2} P_{n-(l-1)}^{\lambda+(l+1)}(\cos \theta) .
$$

By using (3.5) with $n-(l-2)$ and $\lambda+(l-2)$ instead of $n$ and $\lambda$, we can write

$$
\begin{aligned}
& \frac{1}{(n+\lambda)^{l}} \cos \theta(\sin \theta)^{l-2} P_{n-(l-1)}^{\lambda+(l+1)} \\
& =\frac{C(\sin \theta)^{l-2}}{(n+\lambda)^{l-2}}\left(P_{n-(l-2)}^{\lambda+(l-2)}+\frac{C}{(n+\lambda)^{2}} P_{n-(l-2)}^{\lambda+(l-2)}+\frac{C}{(n+\lambda)^{2}}(\sin \theta)^{2} P_{n-l}^{\lambda+l}\right) \\
& =\frac{C(\sin \theta)^{l-2}}{(n+\lambda)^{l-2}} P_{n-(l-2)}^{\lambda+(l-2)}+\frac{C(\sin \theta)^{l-2}}{(n+\lambda)^{l}} P_{n-(l-2)}^{\lambda+(l-2)}+\frac{C(\sin \theta)^{l}}{(n+\lambda)^{l}} P_{n-l}^{\lambda+l} .
\end{aligned}
$$

By Lemma 3.6, the operators are bounded in $L^{2}\left(d m_{\lambda}\right)$.

3.2. Kernel of the Riesz transfom. Observe that by $(1.2), \operatorname{Pf}\left(e^{-t}, \theta\right)=$ $e^{-t \lambda} f\left(e^{-t}, \theta\right)$, where $f(r, \theta)=\sum_{n=0}^{\infty} a_{n} r^{n} P_{n}^{\lambda}(\cos \theta)\left\|P_{n}^{\lambda}\right\|_{L^{2}\left(d m_{\lambda}\right)}^{-1}$ is defined by Muckenhoupt and Stein in $\frac{15}{5}$. They compute explicitly the kernel $P(r, \theta, \phi)$ of 
$f(r, \theta)$ :

$$
P f(r, \theta)=r^{\lambda} \int_{0}^{\pi} P(r, \theta, \phi) f(\phi) d m_{\lambda}(\phi), \quad \theta \in[0, \pi]
$$

where $r=e^{-t}$ and

prtf

$$
P(r, \theta, \phi)=\frac{\lambda}{\pi} \int_{0}^{\pi} \frac{\left(1-r^{2}\right) \sin ^{2 \lambda-1} t}{\left(1-2 r(\cos \theta \cos \phi+\sin \theta \sin \phi \cos t)+r^{2}\right)^{\lambda+1}} d t .
$$

Before continuing further, let us state some useful notation.

notation

$$
\begin{aligned}
& \sigma=\sin \theta \sin \phi, \quad a=\cos \theta \cos \phi+\sigma \cos t=\cos (\theta-\phi)-\sigma(1-\cos t), \\
& \Delta_{r}=1-2 r \cos (\theta-\phi)+r^{2}=(1-r)^{2}+2 r(1-\cos (\theta-\phi)), \\
& \Delta=\Delta_{1}, \quad D_{r}=1-2 r a+r^{2}=\Delta_{r}+2 r \sigma(1-\cos t) .
\end{aligned}
$$

Lemma 3.14 (Lemma 2 in $\frac{\text { BMTU }}{1]}$ ). Given $f \in L^{1}\left(d m_{\lambda}\right)$ and $l \geq 1$, for almost every $\theta \in[0, \pi]$, we have that

$$
\begin{aligned}
& \left(L_{\lambda}\right)^{-\frac{l}{2}} f(\theta)=\int_{0}^{\pi} W_{\lambda}^{l}(\theta, \phi) f(\phi) d m_{\lambda}(\phi), \text { where } \\
& W_{\lambda}^{l}(\theta, \phi)=\frac{1}{\Gamma(l)} \int_{0}^{1} r^{\lambda-1}\left(\log \frac{1}{r}\right)^{l-1} P(r, \theta, \phi) d r .
\end{aligned}
$$

Given $f \in L^{1}\left(d m_{\lambda}\right), l \geq 1$ and $\theta$ outside the support of $f$, we have that

$$
R_{\lambda}^{l} f(\theta)=\int_{0}^{\pi} R_{\lambda}^{l}(\theta, \phi) f(\phi) d m_{\lambda}(\phi), \quad \text { where }
$$

$$
R_{\lambda}^{l}(\theta, \phi)=\frac{1}{\Gamma(l)} \int_{0}^{1} r^{\lambda-1}\left(\log \frac{1}{r}\right)^{l-1} \frac{\lambda}{\pi}\left(1-r^{2}\right) \int_{0}^{\pi}(\sin t)^{2 \lambda-1} \partial_{\theta}^{l}\left(\frac{1}{D_{r}^{\lambda+1}}\right) d t .
$$

Proof. $L_{\lambda}^{-1 / 2}$ is defined in (1.3). Then, to get (3.15) it is enough to apply Fubini's theorem. To prove $(3,16)$, it is enough to justify the differentiation inside the integral sign. See [1] for the details.

Now we shall see that the kernel $R_{\lambda}^{l}(\theta, \phi)$ satisfies the hypothesis of Theorem 2.4. Since $P(r, \pi-\theta, \pi-\phi)=P(r, \theta, \phi)$, then $R_{\lambda}^{l}(\pi-\theta, \pi-\phi)=$ $(-1)^{l} R_{\lambda}^{l}(\theta, \phi)$. Therefore, $R_{\lambda}^{l}(\theta, \phi)$ satisfies condition iii) in Theorem 2.4. Observe that $\partial_{\theta}^{l+1}\left(D_{r}^{-\lambda-1}\right)$ and $\partial_{\phi} \partial_{\theta}^{l}\left(D_{r}^{-\lambda-1}\right)$ are quotients with a bounded function in the numerator and a certain power of $D_{r}$ in the denominator. Since for $r \in(0,1 / 2), D_{r} \geq C$ and for $r \in(1 / 2,1)$ and $|\theta-\phi|>\pi / 6, D_{r} \geq C$, we get

$$
\begin{aligned}
& \left|R_{\lambda}^{l}(\theta, \phi)\right|+\left|\partial_{\theta} R_{\lambda}^{l}(\theta, \phi)\right|+\left|\partial_{\phi} R_{\lambda}^{l}(\theta, \phi)\right| \\
& \quad \leq C \int_{0}^{1 / 2} r^{\lambda-1}\left(\log \frac{1}{r}\right)^{l-1} d r+C \int_{1 / 2}^{1} r^{\lambda-1}\left(\log \frac{1}{r}\right)^{l-1} d r \leq C .
\end{aligned}
$$

With this we obtain condition i) in Theorem 2.4. In order to prove condition ii), we need a careful analysis of $\partial_{\theta}^{l}\left(\frac{1}{D_{r}^{\lambda+1}}\right)$. Recall our notation (3.13) and let 
us call

$$
b=\partial_{\theta} a=-\sin (\theta-\phi)-\cos \theta \sin \phi(1-\cos t)
$$

and observe $\partial_{\theta} b=a$. We have the following lemma to describe $\partial_{\theta}^{l}\left(\frac{1}{D_{r}^{\lambda+1}}\right)$ sum more precisely:

\section{Lemma 3.17.}

$$
\partial_{\theta}^{l}\left(\frac{1}{D_{r}^{\lambda+1}}\right)=\sum c_{l, k, i, j} \frac{r^{i+j} a^{i} b^{j}}{D_{r}^{\lambda+1+k}}
$$

and $c_{l, k, i, j} \neq 0$ only if:

ions derivative

erivative Mlijk

$$
k=1, \ldots, l, \quad i+j=k, \quad j \geq 2 k-l .
$$

Proof. For $l=1, \partial_{\theta}^{l}\left(\frac{1}{D_{r}^{\lambda+1}}\right)=\frac{C r b}{D_{r}^{\lambda+2}}$ and therefore only $c_{1,1,0,1}$ is nonzero. Assume that the lemma is true for $l$. Since

$$
\partial_{\theta}\left(\frac{r^{i+j} a^{i} b^{j}}{D_{r}^{\lambda+1+k}}\right)=c_{1} \frac{r^{i+j} a^{i-1} b^{j+1}}{D_{r}^{\lambda+1+k}}+c_{2} \frac{r^{i+j} a^{i+1} b^{j-1}}{D_{r}^{\lambda+1+k}}+c_{3} \frac{r^{i+j+1} a^{i} b^{j+1}}{D_{r}^{\lambda+1+(k+1)}}
$$

(we assume $c_{1}=0$ if $i=0$ and $c_{2}=0$ if $j=0$ ), we easily see that all these expressions satisfy $(3.18)$ for $l+1$.

As a consequence we may write: $R_{\lambda}^{l}(\theta, \phi)=\sum c_{l, k, i, j} M_{n, k, i, j}(\theta, \phi)$, where $c_{l, k, i, j}$ are as in the last lemma and

$$
M_{l, k, i, j}(\theta, \phi)=\int_{0}^{1} \int_{0}^{\pi} r^{\lambda+i+j-1}\left(\log \frac{1}{r}\right)^{l-1}\left(1-r^{2}\right) \frac{a^{i} b^{j} \sin ^{2 \lambda-1} t}{D_{r}^{\lambda+1+k}} d t d r .
$$

The next step is checking that each of these terms verifies condition ii) of Theorem 2.4. This will be done in two lemmas.

Lemma 3.20. There exists a constant $C>0$ such that, for every $\theta \in[0, \pi / 2]$ and $2 \theta / 3 \leq \phi \leq 3 \theta / 2$, we have

$$
\begin{aligned}
\left|M_{l, k, i, j}(\theta, \phi)\right| & \leq \frac{C}{|\theta-\phi|} \frac{1}{(\sin \phi)^{2 \lambda}} \\
\left|\partial_{\theta} M_{l, k, i, j}(\theta, \phi)\right|+\left|\partial_{\phi} M_{l, k, i, j}(\theta, \phi)\right| & \leq \frac{C}{|\theta-\phi|^{2}} \frac{1}{(\sin \phi)^{2 \lambda}} .
\end{aligned}
$$

Proof. Let us start with the first inequality. Since for $r \in(0,1 / 2), D_{r} \geq C$, and for $r \in(1 / 2,1), \log \frac{1}{r} \leq C(1-r)$, and also by using that $|a| \leq C,|b| \leq$ 
$\sin |\theta-\phi|+\sin \phi(1-\cos t)$, we have

$$
\begin{aligned}
\left|M_{l, k, i, j}(\theta, \phi)\right| & \leq C \int_{0}^{1 / 2} r^{\lambda+k-1}\left(\log \frac{1}{r}\right)^{l-1} d r \\
& +C \int_{1 / 2}^{1} \int_{0}^{\pi}(1-r)^{l} \frac{(\sin |\theta-\phi|+\sin \phi(1-\cos t))^{j}}{D_{r}^{\lambda+k+1}}(\sin t)^{2 \lambda-1} d t d r \\
& \leq C+C \int_{1 / 2}^{1} \int_{0}^{\pi}(1-r)^{l} \frac{(\sin |\theta-\phi|)^{j}(\sin t)^{2 \lambda-1}}{D_{r}^{\lambda+k+1}} d t d r \\
& +C \int_{1 / 2}^{1} \int_{0}^{\pi / 2}(1-r)^{l} \frac{(\sin \phi(1-\cos t))^{j}(\sin t)^{2 \lambda-1}}{D_{r}^{\lambda+k+1}} d t d r \\
& +C \int_{1 / 2}^{1} \int_{\pi / 2}^{\pi}(1-r)^{l} \frac{(\sin \phi)^{j}(\sin t)^{2 \lambda-1}}{D_{r}^{\lambda+k+1}} d t d r=C+I+I I+I I I .
\end{aligned}
$$

For the first term, we use that $j \geq 2 k-l$, and thus $l \geq 2 k-j$, and then we perform successively the changes of variables $x=\sqrt{\frac{\sigma}{\Delta_{r}}} t$ and $u=\frac{1-r}{\sqrt{\Delta}}$.

$$
\begin{aligned}
I & \leq C \int_{1 / 2}^{1}(1-r)^{2 k-j}(\sin |\theta-\phi|)^{j} \int_{0}^{\pi / 2} \frac{t^{2 \lambda-1}}{\left(\Delta_{r}+\sigma t^{2}\right)^{\lambda+k+1}} d t d r \\
& =C \int_{1 / 2}^{1}(1-r)^{2 k-j}(\sin |\theta-\phi|)^{j} \int_{0}^{\frac{\pi}{2} \sqrt{\frac{\sigma}{\Delta_{r}}}} \frac{\left(\sqrt{\frac{\Delta_{r}}{\sigma}} x\right)^{2 \lambda-1} \sqrt{\frac{\Delta_{r}}{\sigma}} d x}{\Delta_{r}^{\lambda+k+1}\left(1+x^{2}\right)^{\lambda+k+1}} d r \\
& \leq(\sin |\theta-\phi|)^{j} C \int_{1 / 2}^{1} \frac{(1-r)^{2 k-j} d r}{\sigma^{\lambda} \Delta_{r}^{k+1}} \\
& \leq C \frac{(\sin |\theta-\phi|)^{j}}{\sigma^{\lambda}} \int_{0}^{\frac{1}{2 \sqrt{\Delta}}} \frac{(\sqrt{\Delta} u)^{2 k-j} \sqrt{\Delta} d u}{\Delta^{k+1}\left(1+u^{2}\right)^{k+1}} d r \\
& \leq C \frac{(\sin |\theta-\phi|)^{j}}{\sigma^{\lambda} \Delta^{j / 2+1 / 2}} \leq \frac{C}{\sigma^{\lambda} \Delta^{1 / 2}} \leq \frac{C}{|\theta-\phi|} \frac{1}{(\sin (\theta \vee \phi))^{2 \lambda}}
\end{aligned}
$$

where in the last two inequalities we have used that for $2 \theta / 3 \leq \phi \leq 3 \theta / 2$,

$$
1-\cos (\theta-\phi) \sim(\sin |\theta-\phi|)^{2}, \quad \sin \theta \sim \sin \phi, \quad|\sin (\theta-\phi)| \leq C \sin \phi
$$

The proof of these inequalities is trivial, although the argument differs when $\phi \in[0, \pi / 2]$ than when $\phi \in[\pi / 2, \pi]$. Analogously we may estimate II and III. 
By (3.19) and analogous arguments as in (3.23), we have

$$
\begin{aligned}
\left|\partial_{\theta} M_{l, k, i, j}(\theta, \phi)\right| \leq & C+C \int_{1 / 2}^{1} \int_{0}^{\pi}(1-r)^{l} \frac{|b|^{j+1}}{D_{r}^{\lambda+k+1}}(\sin t)^{2 \lambda-1} d t d r \\
& +C \int_{1 / 2}^{1} \int_{0}^{\pi}(1-r)^{l} \frac{|b|^{j-1}}{D_{r}^{\lambda+k+1}}(\sin t)^{2 \lambda-1} d t d r \\
& +C \int_{1 / 2}^{1} \int_{0}^{\pi}(1-r)^{l} \frac{|b|^{j+1}}{D_{r}^{\lambda+k+2}}(\sin t)^{2 \lambda-1} d t d r \\
= & C+A+B+D .
\end{aligned}
$$

For the first integral, we have that

$$
A \leq C \int_{1 / 2}^{1} \int_{0}^{\pi}(1-r)^{l} \frac{|b|^{j+1}}{D_{r}^{\lambda+k+1}}(\sin t)^{2 \lambda-1} d t d r \leq \frac{C}{|\theta-\phi|^{2}} \frac{1}{(\sin (\theta \vee \phi))^{2 \lambda}}
$$

as it was proven by the estimates of $I, I I$ and $I I I$ above. Observe that if $j=0$, the second term would not appear. So, we may proceed to estimate $B$ assuming $j \geq 1$, and obtaining

$$
\begin{aligned}
B \leq & C \int_{1 / 2}^{1}(1-r)^{l} \int_{0}^{\pi} \frac{(\sin |\theta-\phi|+\sin \phi(1-\cos t))^{j-1}}{D_{r}^{\lambda+k+1}}(\sin t)^{2 \lambda-1} d t d r \\
\leq & C \int_{1 / 2}^{1} \int_{0}^{\pi / 2}(1-r)^{l} \frac{(\sin |\theta-\phi|)^{j-1}(\sin t)^{2 \lambda-1}}{D_{r}^{\lambda+k+1}} d t d r \\
& +C \int_{1 / 2}^{1} \int_{0}^{\pi / 2}(1-r)^{l} \frac{(\sin \phi(1-\cos t))^{j-1}(\sin t)^{2 \lambda-1}}{D_{r}^{\lambda+k+1}} d t d r \\
& +C \int_{1 / 2}^{1} \int_{\pi / 2}^{\pi}(1-r)^{l} \frac{(\sin \phi)^{j-1}(\sin t)^{2 \lambda-1}}{D_{r}^{\lambda+k+1}} d t d r \\
\leq & \frac{C}{\sigma^{\lambda} \Delta} \leq \frac{C}{|\theta-\phi|^{2}} \frac{1}{(\sin (\theta \vee \phi))^{2 \lambda}},
\end{aligned}
$$

where the last two inequalities follows as in $I, I I$ and $I I I$ above. Observe that

$$
D \leq C \int_{1 / 2}^{1}(1-r)^{l} \int_{0}^{\pi} \frac{(\sin |\theta-\phi|+\sin \phi(1-\cos t))^{j+1}}{D_{r}^{\lambda+k+2}}(\sin t)^{2 \lambda-1} d t d r
$$

The same arguments drive to the bound

$$
D \leq \frac{C}{|\theta-\phi|^{2}} \frac{1}{(\sin (\theta \vee \phi))^{2 \lambda}}
$$

The proof of (3.22) for $\partial_{\phi}$ follows the same lines.

Lemma 3.25. For every $\theta, \phi \in[0, \pi / 2]$ and $\phi$ outside the region $2 \theta / 3 \leq \phi \leq$ $3 \theta / 2$, we also have (3.21) and (3.22).

Proof. In the following calculations we will use that for $\theta, \phi \in[0, \pi / 2]$, then $1-\cos (\theta-\phi) \sim|\theta-\phi|^{2} \sim(\sin |\theta-\phi|)^{2}$. Also, for $\phi$ outside the region 
$2 \theta / 3 \leq \phi \leq 3 \theta / 2, \sin |\theta-\phi| \sim \sin (\theta \vee \phi)$ and $|b| \leq C \sin |\theta-\phi|$. Therefore, by the same techniques applied in $(3.23)$, for $M_{l, k, i, j}(\theta, \phi)$ we have

$$
\begin{aligned}
\left|M_{l, k, i, j}(\theta, \phi)\right| & \leq C+C \int_{1 / 2}^{1}(1-r)^{2 k-j} \frac{|b|^{j}}{\Delta_{r}^{\lambda+k+1}} d r \\
& \leq C+C(\sin |\theta-\phi|)^{j} \int_{1 / 2}^{1} \frac{(1-r)^{2 k-j}}{\Delta_{r}^{\lambda+k+1}} d r \\
& \leq C+C(\sin |\theta-\phi|)^{j} \int_{0}^{\frac{1}{2 \sqrt{\Delta}}} \frac{(\sqrt{\Delta} u)^{2 k-j} \sqrt{\Delta} d u}{\Delta^{\lambda+k+1}\left(1+u^{2}\right)^{\lambda+k+1}} d r \\
& \leq C+C \frac{(\sin |\theta-\phi|)^{j}}{\Delta^{\lambda+j / 2+1 / 2}} \leq \frac{C}{|\theta-\phi|} \frac{1}{(\sin (\theta \vee \phi))^{2 \lambda}}
\end{aligned}
$$

We proceed analogously for the derivative

$$
\begin{aligned}
& \left|\partial_{\theta} M_{l, k, i, j}(\theta, \phi)\right| \leq C+C \int_{1 / 2}^{1} \int_{0}^{\pi}(1-r)^{l} \frac{|b|^{j+1}}{D_{r}^{\lambda+k+1}}(\sin t)^{2 \lambda-1} d t d r \\
& +C \int_{1 / 2}^{1} \int_{0}^{\pi}(1-r)^{l} \frac{|b|^{j-1}}{D_{r}^{\lambda+k+1}}(\sin t)^{2 \lambda-1} d t d r \\
& +C \int_{1 / 2}^{1} \int_{0}^{\pi}(1-r)^{l} \frac{|b|^{j+1}}{D_{r}^{\lambda+k+2}}(\sin t)^{2 \lambda-1} d t d r \leq \frac{C}{|\theta-\phi|^{2}} \frac{1}{(\sin (\theta \vee \phi))^{2 \lambda}} .
\end{aligned}
$$

on g function $r$

equation $\mathrm{Tg} 1$

$\mathrm{n}$ bddness of $\mathrm{g}$

\section{Proof of Theorem 1.8}

Let us write $\mathcal{G}^{i} f(\theta)=\left\|T_{\mathcal{G}^{i}} f(\theta)\right\|_{L^{2}((0,1), d r)}, i=1,2$ where $T_{\mathcal{G}^{i}}$ is the operator mapping a scalar valued functions into $L^{2}(d r)$-valued functions given by

$$
T_{\mathcal{G}^{1}} f(\theta)=\sqrt{r \log \frac{1}{r}} \partial_{r} \operatorname{Pf}(r, \theta), \quad T_{\mathcal{G}^{2}} f(\theta)=\sqrt{r \log \frac{1}{r}} \partial_{\theta} \operatorname{P} f(r, \theta) .
$$

4.1. Boundedness in $L^{2}\left(d m_{\lambda}\right)$ of the $g$-functions. For any polynomial function $f \in L^{2}\left(d m_{\lambda}\right)$, the operator $\partial_{r} P f=\sum_{n=0}^{N}(n+\lambda) r^{n+\lambda-1} a_{n} P_{n}^{\lambda}\left\|P_{n}^{\lambda}\right\|^{-1}$ gives a well defined function in $L^{2}\left(d m_{\lambda}\right)$, since for each fixed $r \in(0,1),\left|(n+\lambda) r^{n+\lambda}\right| \leq$ $C$ for any $n \geq 0$. Thus, we can write

$$
T_{\mathcal{G}^{1}} f=\sum_{n=0}^{\infty} \sqrt{r \log \frac{1}{r}}(n+\lambda) r^{n+\lambda-1} a_{n} P_{n}^{\lambda}\left\|P_{n}^{\lambda}\right\|^{-1}=\sum_{n=0}^{\infty} g^{1}(n) a_{n} P_{n}^{\lambda}\left\|P_{n}^{\lambda}\right\|^{-1},
$$

where $g^{1}(n)=\sqrt{r \log \frac{1}{r}}(n+\lambda) r^{n+\lambda-1}$ belongs to $L^{2}(d r)$ uniformly in $n$. In particular, this implies that $\mathcal{G}^{1}$ is bounded in $L^{2}$. To get the boundedness of $\mathcal{G}^{2}$ in $L^{2}$ we proceed similarly. Observe that for any polynomial $f \in L^{2}\left(d m_{\lambda}\right)$,

$$
\partial_{\theta} P f=\sum_{n=1}^{N} r^{n+\lambda} a_{n}(-2 \lambda) \sin \theta P_{n-1}^{\lambda+1}\left\|P_{n}^{\lambda}\right\|^{-1}
$$


We can write

$$
\begin{aligned}
T_{\mathcal{G}^{2}} f & =\sum_{n=1}^{N} \sqrt{r \log \frac{1}{r}} r^{n+\lambda} \frac{a_{n}}{n+\lambda}(-2 \lambda) \sin \theta P_{n-1}^{\lambda+1}\left\|P_{n}^{\lambda}\right\|^{-1} \\
& =\sum_{n=0}^{\infty} g^{2}(n) \frac{a_{n}}{n+\lambda}(-2 \lambda) \sin \theta P_{n-1}^{\lambda+1}\left\|P_{n}^{\lambda}\right\|^{-1}=T_{g^{2}}\left(R_{\lambda} f\right),
\end{aligned}
$$

where $R_{\lambda}$ is the Riesz transform operator (see [1] and $T_{g^{2}}$ is the multiplier associated to the orthogonal system in $L^{2}\left(d m_{\lambda}\right)$ given by the functions $h_{n}(\theta)=$ $\sin \theta P_{n-1}^{\lambda+1}(\cos \theta)$ with coefficients $g^{2}(n)=\sqrt{r \log \frac{1}{r}}(n+\lambda) r^{n+\lambda-1}$. The coefficients are uniformly bounded in $L_{2}^{2}(d r)$. This, together with the $L^{2}$-boundedness of the Riesz transform, see [1] for the details, give that $\mathcal{G}^{2}$ is bounded in $L^{2}\left(d m_{\lambda}\right)$.

4.2. Kernel of the $g$-function. In the next lemma, we find the vector-valued kernel in the Calderón-Zygmund sense of $T_{\mathcal{G}^{i}}, i=1,2$.

hel in $\mathrm{CZ}$ sense

equation $\operatorname{Tg} 2$

Lemma 4.2. For every $f \in L^{1}\left(d m_{\lambda}\right)$ and $\theta$ outside the support of $f$, we have

$$
T_{\mathcal{G}^{i}} f(\theta)=\int_{0}^{\pi} \tau_{r}^{i}(\theta, \phi) f(\phi) d m_{\lambda}(\phi), \quad i=1,2,
$$

where

$$
\begin{aligned}
\tau_{r}^{1}(\theta, \phi)=\sqrt{r \log \frac{1}{r}} \frac{\lambda}{\pi}\left[\lambda r^{\lambda-1} P(r, \theta, \phi)-2 r^{\lambda+1} \int_{0}^{\pi} \frac{(\sin t)^{2 \lambda-1}}{D_{r}^{\lambda+1}} d t\right. \\
\left.-(\lambda+1) r^{\lambda}\left(1-r^{2}\right) \int_{0}^{\pi} \frac{(\sin t)^{2 \lambda-1} \partial_{r} D_{r}}{D_{r}^{\lambda+2}} d t\right] \\
(4.4) \tau_{r}^{2}(\theta, \phi)=\sqrt{r \log \frac{1}{r} \frac{\lambda(\lambda+1)}{\pi} r^{\lambda}\left(1-r^{2}\right) \int_{0}^{\pi} \frac{(\sin t)^{2 \lambda-1} \partial_{\theta} D_{r}}{D_{r}^{\lambda+2}} d t}
\end{aligned}
$$

are kernels taking values in $L^{2}(d r)$.

Proof. By the definition of $T_{\mathcal{G}^{i}}$, in order to get (4.3) we only need to put the derivative inside the integrals in the expressions (4.1). With our usual notation (3.13), we write

$$
\partial_{r} P(r, \theta, \phi)=-\frac{\lambda}{\pi} 2 r \int_{0}^{\pi} \frac{(\sin t)^{2 \lambda-1}}{D_{r}^{\lambda+1}} d t+\frac{\lambda}{\pi}\left(1-r^{2}\right) \partial_{r} \int_{0}^{\pi} \frac{(\sin t)^{2 \lambda-1}}{D_{r}^{\lambda+1}} d t
$$

For negative $a, D_{r} \geq 1$, and for positive $a, a \leq \cos (\theta-\phi)$. Therefore, if $\theta$ does not belong to the support of $f$, we have that $D_{r} \geq 1-\cos (\theta-\phi)^{2} \geq C$. This implies that for each $r$ the remaining integrands in the right hand of the equations in (4.1) belong to $L^{1}\left(d m_{\lambda} \times d t\right)$ for $f \in L^{1}\left(d m_{\lambda}\right)$. This shows (4.4). Also, if $\theta$ does not belong to the support of $f$, we have that $\left|\tau_{r}^{1}(\theta, \phi)\right| \leq$ $C \sqrt{r \log \frac{1}{r}} r^{\lambda-1}$ and $\left|\tau_{r}^{2}(\theta, \phi)\right| \leq C$, which are functions in $L^{2}(d r)$. 
Our aim is to prove that the kernels of $T_{\mathcal{G}^{1}}$ verify the hypothesis of Theorem 2.4. We have already seen that they are bounded in $L^{2}\left(d m_{\lambda}\right)$. Observe that since $P(r, \pi-\theta, \pi-\phi)=P(r, \theta, \phi)$, then the symmetry condition iii) holds.

On the other hand, for $|\theta-\phi|>\pi / 6$ and $r \in(1 / 2,1)$, then $D_{r} \geq 1-$ $(\cos (\theta-\phi))^{2} \geq C$. For $r \in(0,1 / 2)$, clearly $D_{r} \geq C$. Then $\left\|\tau_{r}^{1}(\theta, \phi)\right\|_{L^{2}(d r)} \leq$ $C\left\|\sqrt{r \log \frac{1}{r}}\left(r^{\lambda-1}+1\right)\right\|_{L^{2}(d r)}=C$ and $\left\|\tau_{r}^{2}(\theta, \phi)\right\|_{L^{2}(d r)} \leq C$, as can be easily seen from (4.4) and (4.5). Thus condition i) in Theorem 2.4 also holds.

Checking condition ii) requires a bit more of work.

local estimates

l estimate g 1

al estimate g 2

Lemma 4.7. There exists a constant $C>0$ such that, for every $\theta \in[0, \pi / 2]$ and $2 \theta / 3 \leq \phi \leq 3 \theta / 2$, we have (for $i=1,2$ )

$$
\begin{aligned}
\left\|\tau_{r}^{i}(\theta, \phi)\right\|_{L^{2}(d r)} & \leq \frac{C}{|\theta-\phi|} \frac{1}{(\sin (\theta \vee \phi))^{2 \lambda}} \\
\left.\left\|\partial_{\theta} \tau_{r}^{i}(\theta, \phi)\right\|_{L^{2}(d r)}+\| \partial_{\phi} \tau_{r}^{i}(\theta, \phi)\right] \|_{L^{2}(d r)} & \leq \frac{C}{|\theta-\phi|^{2}} \frac{1}{(\sin (\theta \vee \phi))^{2 \lambda}}
\end{aligned}
$$

Proof. When $r \in(0,1 / 2), D_{r} \geq(1-r)^{2} \geq C$ and therefore $\left|\tau_{r}^{1}(\theta, \phi)\right| \leq$ $C \sqrt{r \log \frac{1}{r}}\left(r^{\lambda-1}+C\right)$ and $\left|\tau_{r}^{2}(\theta, \phi)\right| \leq C$. For $r \in(1 / 2,1)$, let us observe that, by (4.4), we can split $\left|\tau_{r}^{1}(\theta, \phi)\right|$ according to the following sum $\left|\partial_{r}\left(r^{\lambda} P(r, \theta, \phi)\right)\right| \leq$ $\sum_{i=1}^{3} N_{i}(r, \theta, \phi)$, where

$$
\begin{gathered}
N_{1}(r, \theta, \phi)=C \int_{0}^{\pi / 2} \frac{(\sin t)^{2 \lambda-1}}{D_{r}^{\lambda+1}} d t, \quad N_{2}(r, \theta, \phi)=C \int_{\pi / 2}^{\pi} \frac{(\sin t)^{2 \lambda-1}}{D_{r}^{\lambda+1}} d t, \\
N_{3}(r, \theta, \phi)=C(1-r) \int_{0}^{\pi} \frac{(\sin t)^{2 \lambda-1}\left|\partial_{r} D_{r}\right|}{D_{r}^{\lambda+2}} d t
\end{gathered}
$$

We will use the following estimate

ialphabeta

$$
I_{\lambda+1}^{2 \lambda-1}=\int_{0}^{\frac{\pi}{2}} \frac{t^{2 \lambda-1}}{\left(\Delta_{r}+r \sigma t^{2}\right)^{\lambda+1}} d t \leq \frac{C}{\Delta_{r} r^{\lambda} \sigma^{\lambda}},
$$

which can be easily obtained by the change of variables $t=\Delta_{r}^{\frac{1}{2}}(r \sigma)^{-\frac{1}{2}} u$. By using that $\sin x \sim x, 1-\cos x \sim x^{2}$ and $1+\cos x \geq C x^{2}$ for $x \in[0, \pi / 2]$, it is not difficult to obtain the following estimate,

$$
N_{1}(r, \theta, \phi)+N_{2}(r, \theta, \phi) \leq C I_{\lambda+1}^{2 \lambda-1},
$$

where in the case of $N_{2}$ we have firstly made the change of variables $\pi-x=t$. For the term $N_{3}$, observe that for $r \in[1 / 2,1]$

partial dr

$$
(1-r)\left|\partial_{r} D_{r}\right| \leq C(1-r)[|1-r|+|1-\cos (\theta-\phi)|+|\sigma(1-\cos t)|] \leq C D_{r}
$$

Thus, after applying the same change of variables $\pi-x=t$ used above for $N_{2}$, we get that $N_{3}(r, \theta, \phi) \leq C I_{\lambda+1}^{2 \lambda-1}$. This gives (and observe that with the only restriction $r \in[1 / 2,1])$ that

$$
\left|\partial_{r}\left(r^{\lambda} P(r, \theta, \phi)\right)\right| \leq C I_{\lambda+1}^{2 \lambda-1} .
$$


Next step is integrating in $r$. By using (4.10), $\Delta_{r} \geq C\left((1-r)^{2}+\Delta\right), r \log \frac{1}{r} \sim$ $1-r$ for $r \in(1 / 2,1)$, the change of variables $u=1-r / \sqrt{\Delta}$ and (3.24), we obtain

$$
\int_{1 / 2}^{1}\left|\tau_{r}^{1}(\theta, \phi)\right|^{2} d r \leq \frac{C}{\sigma^{2 \lambda}} \int_{1 / 2}^{1} \frac{r \log \frac{1}{r} d r}{\left((1-r)^{2}+\Delta\right)^{2}} \leq \frac{C}{\sigma^{2 \lambda} \Delta} \leq \frac{C}{|\theta-\phi|^{2}(\sin (\theta \vee \phi))^{4 \lambda}}
$$

The case of $\tau_{r}^{2}$ is treated similarly. For the estimates concerning the derivative, let us observe first that the same arguments as in Lemma 4.2 allow us to put the derivatives inside the integrals, and then

ivative th tau1

partial th dr

$$
\begin{aligned}
& \text { (4.13) } \partial_{\theta} \tau_{r}^{1}(\theta, \phi)=\sqrt{r \log \frac{1}{r}} \frac{\lambda}{\pi}\left[r^{\lambda-1}\left(\lambda\left(1-r^{2}\right)-r^{2}\right) \int_{0}^{\pi} \frac{(\sin t)^{2 \lambda-1} \partial_{\theta} D_{r}}{D_{r}^{\lambda+2}} d t\right. \\
& \left.-(\lambda+1) r^{\lambda}\left(1-r^{2}\right) \int_{0}^{\pi} \frac{(\sin t)^{2 \lambda-1}}{D_{r}^{\lambda+1}} \frac{\partial_{\theta} \partial_{r} D_{r} D_{r}-(\lambda+2) \partial_{\theta} D_{r} \partial_{r} D_{r}}{D_{r}^{2}} d t\right] d r,
\end{aligned}
$$

By using (4.11), its analogous $\left|\frac{(1-r) \partial_{\theta} D_{r}}{D_{r}}\right| \leq C,\left|\partial_{\theta}^{2} D_{r}\right| \leq C$ and also the following estimates for $1 / 2 \leq r \leq 1$,

$$
\begin{aligned}
& \left|\frac{\partial_{\theta} D_{r}}{D_{r}}\right| \leq \frac{2 r \sin |\theta-\phi|}{2 r(1-\cos (\theta-\phi))}+\frac{2 r \cos \theta \sin \phi(1-\cos t)}{2 r \sin \theta \sin \phi(1-\cos t)} \leq \frac{C}{|\theta-\phi|} \\
& \left|\frac{\partial_{\theta} \partial_{r} D_{r}}{D_{r}}\right|=\left|\frac{1 / r \partial_{\theta} D_{r}}{D_{r}}\right| \leq \frac{C}{|\theta-\phi|}
\end{aligned}
$$

we easily get that

$$
\left|\partial_{\theta} \tau_{r}^{1}(\theta, \phi)\right| \leq \frac{C}{\sigma^{\lambda}|\theta-\phi|} \sqrt{r \log \frac{1}{r}}\left(r^{\lambda-1} 1_{(0,1 / 2)}(r)+I_{\lambda+1}^{2 \lambda-1} 1_{(1 / 2,1)}(r)\right),
$$

and therefore, for $\theta$ and $\phi$ satisfying $2 \theta / 3 \leq \phi \leq 3 \theta / 2$, we have

$$
\left\|\partial_{\theta} \tau_{r}^{1}(\theta, \phi)\right\|_{L^{2}(d r)} \leq \frac{C}{|\theta-\phi|^{2}} \frac{1}{(\sin (\theta \vee \phi))^{2 \lambda}} .
$$

The derivative in $\phi$ is treated in the same way, by using the parallel estimates to (4.11) and (4.14). Also, for $\tau_{r}^{2}$ similar arguments hold.

Lemma 4.15. For every $\theta, \phi \in[0, \pi / 2]$ and $\phi$ outside the region $2 \theta / 3 \leq \phi \leq$ $3 \theta / 2$, we also have (4.8) and (4.9).

Proof. We use that from (4.12) one can achieve the following inequality

$$
\left|\tau_{r}^{1}(\theta, \phi)\right| \leq C \sqrt{r \log \frac{1}{r}}\left(r^{\lambda-1} 1_{(0,1 / 2)}(r)+I_{\lambda+1}^{2 \lambda-1} 1_{(1 / 2,1)}(r)\right),
$$

and using that for $r \in(1 / 2,1), r \log \frac{1}{r} \sim 1-r$, we get

au_r i estimate

$$
\begin{aligned}
\left\|\tau_{r}^{1}(\theta, \phi)\right\|_{L^{2}(d r)}^{2} & \leq C+C \int_{1 / 2}^{1} \frac{r \log \frac{1}{r}}{\Delta_{r}^{2(\lambda+1)}} d r \\
& \leq C+C \int_{1 / 2}^{1} \frac{1-r}{\left((1-r)^{2}+\Delta\right)^{2(\lambda+1)}} d r, \leq \frac{C}{\Delta^{2 \lambda+1}}
\end{aligned}
$$


where the last estimate can be easily obtained by the change of variable $u=\frac{1-r}{\sqrt{\Delta}}$. For $\phi<2 \theta / 3, \sin (\theta-\phi) \sim \sin \theta$ and $1-\cos (\theta-\phi) \sim|\theta-\phi|^{2} \sim \sin (\theta-\phi)^{2}$. Thus, by these properties, (4.16) implies

$$
\left\|\tau_{r}^{1}(\theta, \phi)\right\|_{L^{2}(d r)} \leq \frac{C}{(1-\cos (\theta-\phi))^{\lambda+1 / 2}} \leq \frac{C}{|\theta-\phi|} \frac{C}{(\sin (\theta \vee \phi))^{2 \lambda}} .
$$

Now, if $\phi>3 \theta / 2$, then $1-\cos (\theta-\phi) \sim|\theta-\phi|^{2} \sim(\sin (\theta-\phi))^{2}$ and also $|\sin (\theta-\phi)| \sim \sin \phi$. Then, from (4.16) we get as before

$$
\left\|\tau_{r}^{1}(\theta, \phi)\right\|_{L^{2}(d r)} \leq \frac{C}{|\theta-\phi|} \frac{C}{(\sin (\theta \vee \phi))^{2 \lambda}}
$$

The estimate for $\tau_{r}^{2}$ follows in an analogous way. We will obtain (4.9) for the derivative in $\theta$ of $\tau_{r}^{1}$. The proof for the other derivative is completely analogous, and also for $\tau_{r}^{2}$. Let us observe that for $r \in(0,1 / 2)$, then $D_{r} \geq C$ and therefore $\left|\partial_{\theta} \tau_{r}^{i}(\theta, \phi)\right| \leq C \sqrt{r \log \frac{1}{r}}\left(r^{\lambda-1}+1\right)$. If $r \in(1 / 2,1)$, it is easy to see that

n estimates dr

$$
\begin{gathered}
\left|\partial_{\theta} D_{r}\right|,\left|\partial_{\theta} \partial_{r} D_{r}\right| \leq C(\sin |\theta-\phi|+\sin \phi) \\
\left|\partial_{\theta}^{2} D_{r}\right| \leq C, \quad\left|\partial_{\theta} D_{r}\right|^{2} \leq C\left((\sin |\theta-\phi|)^{2}+(\sin \phi)^{2}\right) .
\end{gathered}
$$

Thus, from $(4.13),(4.11)$ and (4.17), it is not difficult to obtain that

$$
\left|\partial_{\theta} \tau_{r}^{1}(\theta, \phi)\right| \leq C \sqrt{r \log \frac{1}{r}}\left(r^{\lambda-1}+1\right) \chi_{(0,1 / 2)}(r)+C \sqrt{r \log \frac{1}{r} \frac{\sin |\theta-\phi|+\sin \phi}{\Delta_{r}^{\lambda+2}}}
$$

From here,

$$
\left\|\partial_{\theta} \tau^{1}(\theta, \phi)\right\|_{L^{2}(d r)} \leq C+C(\sin |\theta-\phi|+\sin \phi) A
$$

where

$$
A^{2}=\int_{1 / 2}^{1} \frac{r \log \frac{1}{r}}{\left((1-r)^{2}+\Delta\right)^{2 \lambda+4}} d r \leq \frac{C}{\Delta^{2 \lambda+3}} .
$$

In the former inequality we have used that for $r \in(1 / 2,1)$ then $r \log \frac{1}{r} \sim 1-r$ and we have performed the change of variables $u=(1-r) / \sqrt{\Delta}$. Thus, we obtain

$$
\left\|\partial_{\theta} \tau^{1}(\theta, \phi)\right\|_{L^{2}(d r)} \leq C+C \frac{\sin |\theta-\phi|+\sin \phi}{\Delta^{\lambda+3 / 2}}
$$

In the region $\phi<2 \theta / 3$, we have $|\theta-\phi| \sim \sin |\theta-\phi| \sim \sin \theta$, and $\Delta \sim$ $(\sin |\theta-\phi|)^{2}$. Thus,

$$
\left\|\partial_{\theta} \tau^{1}(\theta, \phi)\right\|_{L^{2}(d r)} \leq C+C \frac{|\theta-\phi|}{\Delta^{3 / 2}} \frac{1}{\Delta^{\lambda}} \leq \frac{C}{|\theta-\phi|^{2}} \frac{1}{(\sin (\theta \vee \phi))^{2 \lambda}}
$$

On the other hand, for $\phi>3 \theta / 2,|\theta-\phi| \sim \sin |\theta-\phi| \sim \sin \phi$, and $\Delta \sim$ $(\sin |\theta-\phi|)^{2}$. Thus, we obtain the desired estimate in the same way. 
4.3. Muckhenhoupt-Stein $g$-function. The $g$-function defined by Muckhenhoupt and Stein in $[5]$ associated to the ultraspherical polynomials is

function of MS

$$
g f(\theta)=\left(\int_{0}^{1}(1-r)\left|\partial_{r} f(r, \theta)\right|^{2} d r\right)^{1 / 2}
$$

where $f(r, \theta)$ is described in subsection 3.2. They obtain that this operator is bounded in $L^{p}\left(d m_{\lambda}\right)$ for every $p \in(1, \infty)$. A natural question is whether this operator can be handled with the technique developed in this paper. In other words, we would like to see if the operator can be described as a CalderónZygmund operator. It turns out that our computations hold for the operator

$$
g_{1} f(\theta)=\left(\int_{1 / 2}^{1}(1-r)\left|\partial_{r} f(r, \theta)\right|^{2} d r\right)^{1 / 2}
$$

The remaining part

$$
g_{0} f(\theta)=\left(\int_{0}^{1 / 2}(1-r)\left|\partial_{r} f(r, \theta)\right|^{2} d r\right)^{1 / 2}
$$

is easier to handle, and one easily gets that $g_{0}$ maps $L^{p}\left(d m_{\lambda}\right)$ into $L^{p}\left(d m_{\lambda}\right)$ for every $p \in[1, \infty]$.

\section{REFERENCES}

BMTU [1] Buraczewski, D., Martínez, T., Torrea, J.L., Urban, R., On the Riesz transform for ultraspherical expansions, preprint.

Cal [2] Calderón, A. P., Inequalities for the maximal function relative to a metric, Studia Math. 57 (1976), 297-306.

C [3] Christ, M., Lectures on singular integral operators, CBMS Regional Conference Series in Mathematics, 77. Published for the Conference Board of the Mathematical Sciences, Washington DC, by the American Mathematical Society, Providence, RI, 1990.

[4] Coifman, R., Weiss, G., Analyse Harmonique non commutative sur certains espaces homogenes, Lecture Notes in Mathematics 242, Springer-Verlag, Berlín, 1971.

[5] Muckenhoupt, B., Stein, E.M., Classical expansions and their relation to conjugate harmonic functions, Trans. Amer. Math. Soc., 118 (1965), 17-92.

RT [6] Ruiz, F.J., Torrea, J.L., Vector-valued Calderón-Zygmund theory and Carleson measures on spaces of homogeneous nature Studia Math. 88 (1988), 221-243.

st1 [7] Stein, E.M., Topics in Harmonic Analysis Related to the Littlewood-Paley Theory, Princeton University Press and the University of Tokio Press, Princeton, New Jersey, 1970.

[8] Szegö, G., Orthogonal Polynomials, American Mathematical Society Colloquium Publications, v. 23, American Mathematical Society, New York, 1939. 
Institute of Mathematics, Wroclaw University, Plac Grunwaldzki 2/4, 50384 Wroclaw, Poland

E-mail address: dbura@math.uni.wroc.pl

Departamento de Matemáticas, Faculdad de Ciencias, Universidad AutóNOMA DE MADRID, 28049 MADRID, SPAIN

E-mail address: teresa.martinez@uam.es

SAME ADDRESS IN MADRID

E-mail address: joseluis.torrea@uam.es 